\title{
Analyse des Trag- und Verformungsverhaltens von Stahlfaserbetontübbingen für den maschinellen Tunnelbau
}

\author{
Paul Gehwolf, Robert Wenighofer und Robert Galler
}

Lehrstuhl für Subsurface Engineering, Department Mineral Resources Engineering, Montanuniversität Leoben, Leoben, Österreich

Eingegangen 2. November 2018; angenommen 5. November 2018; online publiziert 14. November 2018

\begin{abstract}
Zusammenfassung: Bei internationalen Tunnelprojekten mit maschinellen Vortrieben wird für die Produktion der Tübbinge immer häufiger (Stahl-)Faserbeton für den Ersatz der konventionellen Bewehrungskörbe eingesetzt. Aufgrund des aktuellen Stands der Regelwerke und Richtlinien ist der Einsatz von Stahlfaserbetontübbingen im Tunnelbau in Österreich nicht zulässig und nur Stahlbetontübbinge mit konventionellem Bewehrungskorb dürfen eingesetzt werden. Zur Verifizierung des Trag- und Verformungsverhaltens dieser Faserbetonsegmente wurden Labor- sowie Großversuche am Lehrstuhl für Subsurface Engineering durchgeführt. Wie im BHM 12/2015 berichtet, wurde in Kooperation der ÖBB Infrastruktur AG und der Montanuniversität Leoben - Lehrstuhl für Subsurface Engineering ein Tübbingprüfstand zur Untersuchung dieser Betonfertigteile unter Laborbedingungen errichtet. Auf deren Basis wurden die Anwendung gängiger Dimensionierungsregelwerke überprüft und auftretende Unzulänglichkeiten und deren Gründe aufgezeigt.
\end{abstract}

Schlüsselwörter: Faserbeton, Tübbinge, Tübbingprüfstand

Analyses of the Load Bearing and Deformation Behaviour of Steel Fibre Reinforced Segmental Lining for Mechanized Tunnelling

Abstract: At many international tunnelling projects with shield tunnelling the conventional reinforcement cage is replaced by the usage of (steel) fibre reinforced concrete for the production of segmental lining more and more frequently. Due to the current status of regulations and guidelines the application of steel fibre reinforced segmental lining is in Austria ineligible and only conventional reinforce-

Dipl.-Ing. Dr. mont. P. Gehwolf $(\square)$

Lehrstuhl für Subsurface Engineering, Department Mineral

Resources Engineering,

Montanuniversität Leoben,

Franz-Josef-Straße 18,

8700 Leoben, Österreich

paul.gehwolf@unileoben.ac.at ment cages are allowed. For the verification of the load bearing and deformation behaviour of fibre reinforced segments laboratory and big-scale tests were conducted. As published in BHM 12/2015, a test-rig for investigations under laboratory conditions for these precast elements was built in cooperation between the Austrian Federal Railways and the Montanuniversity Leoben - Chair of Subsurface Engineering. Based on the results the application of current design models was checked and inadequacies were pointed out.

Keywords: Fibre concrete, Segmental lining, Tubbing testrig

\section{Einleitung}

Die Verwendung von konventionell bewehrten Stahlbetonfertigteilen stellt im maschinellen Tunnelbau den Stand der Technik seit vielen Jahrzehnten dar, der durch ständige Weiterentwicklung sehr hohe Qualitäten aufweist. Zur Optimierung der Produktion dieser Segmente wird in letzter Zeit bei internationalen Projekten immer häufiger (Stahl-)Faserbeton für den Ersatz des Bewehrungskorbs herangezogen. Der große Vorteil von Stahlfaserbeton mit statisch wirksamen Makrofasern gegenüber Stahlbeton ist, dass die Fasern unter Zugbelastung im Nachbruchbereich des Betons die freiwerdenden Kräfte aufnehmen können und somit die herkömmliche Stabbewehrung unter Umständen entfallen kann. Hierdurch ergeben sich maßgebliche Vorteile hinsichtlich des Wegfalls der Produktion und Bereithaltung der meist sehr aufwändigen Bewehrungskörbe sowie der Vereinfachung der Arbeitsschritte im Tübbingwerk.

Die Aktivierung der Fasern nach Auftreten des Erstrisses bei Zugbeanspruchung führt, verglichen zu Normalbeton, zu einer erhöhten Restzugfestigkeit in Abhängigkeit des Fasergehalts (Abb. 1). Je nach Faserwerkstoff und Fasertyp erfolgt diese Aktivierung durch den Auszug der Faser aus der Betonmatrix oder bei sehr starker Endverankerung durch Beanspruchung der Faser bis zum Bruch. 
$\sigma_{\mathrm{t}}$

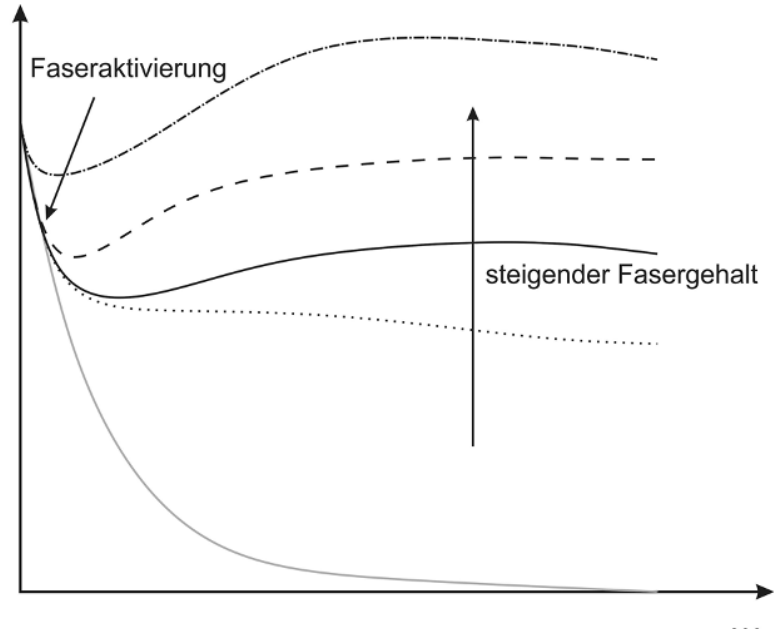

W

Abb. 1: Zugspannung-Rissöffnungsdiagramm für Normalbeton (grau) und Faserbeton bei steigendem Fasergehalt (schwarz) [2]
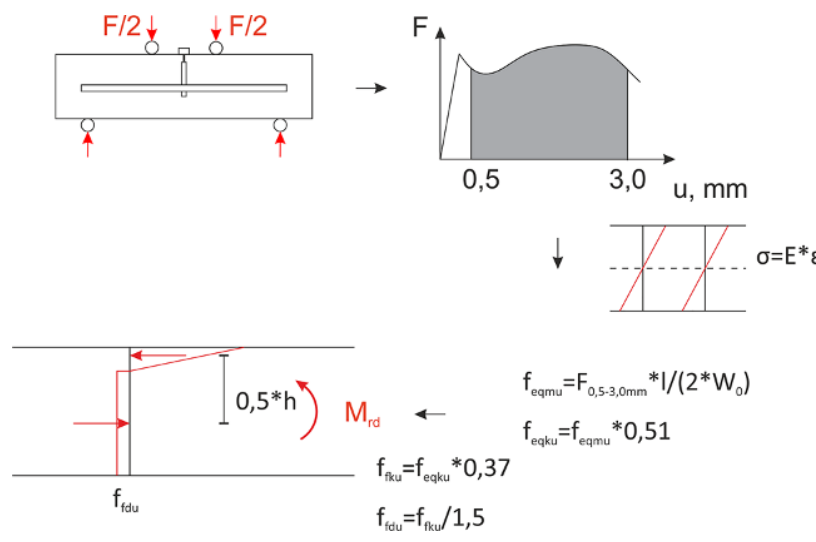

Abb. 2: Bemessungsablauf nach ÖVBB-RL „Faserbeton“ [4]

\section{Bemessung Stahlfaserbeton}

In Österreich erfolgt die Ermittlung der Einwirkungen auf den Tübbingausbau anhand der Richtlinie „Tübbingsysteme aus Beton" [3] der österreichischen Bautechnikvereinigung und die Bemessung der Bauteile auf Basis der Richtlinie "Faserbeton" [4]. Die Werkstoffparameter zur Beschreibung des Zugverhaltens werden mithilfe von Vierpunktbiegeversuchen mit Balken mit einem Querschnitt von $15 \times 15 \mathrm{~cm}^{2}$ ermittelt.

Für die Ermittlung des Grenzzustands der Tragfähigkeit (Abb. 2) eines Bauteils nach ÖVBB-RL [4] sind mindestens sechs Vierpunktbiegeversuche erforderlich. Aus diesen Versuchen werden der Mittelwert und der Varianzkoeffizient ermittelt. Bei Varianzkoeffizienten kleiner als 0,25 wird die äquivalente Biegezugfestigkeit $f_{\text {eqmu }}$ mit linear-elastischem Ansatz und dem ungerissenen Widerstandsmoment $W_{0}$ über den gesamten Bereich der Durchbiegung von 0,5 bis $3,0 \mathrm{~mm}$ aus der Mittelwertkurve gebildet. Um dafür den charakteristischen Wert $\mathrm{f}_{\text {eqku }}(5 \%$-Fraktile) zu erhalten, wird die mittlere äquivalente Biegezugfestigkeit $f_{\text {eqmu }}$ mit 0,51 multipliziert. Diese charakteristische Biegezugfestigkeit wird

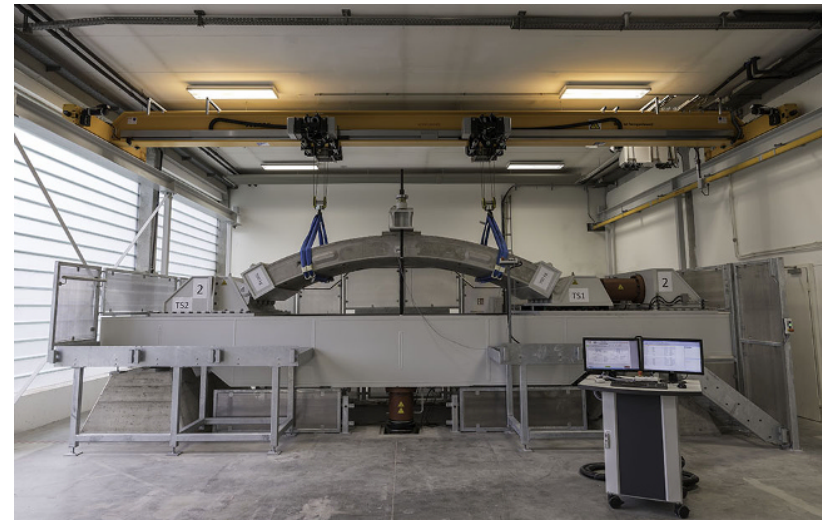

Abb. 3: Tübbingprüfstand
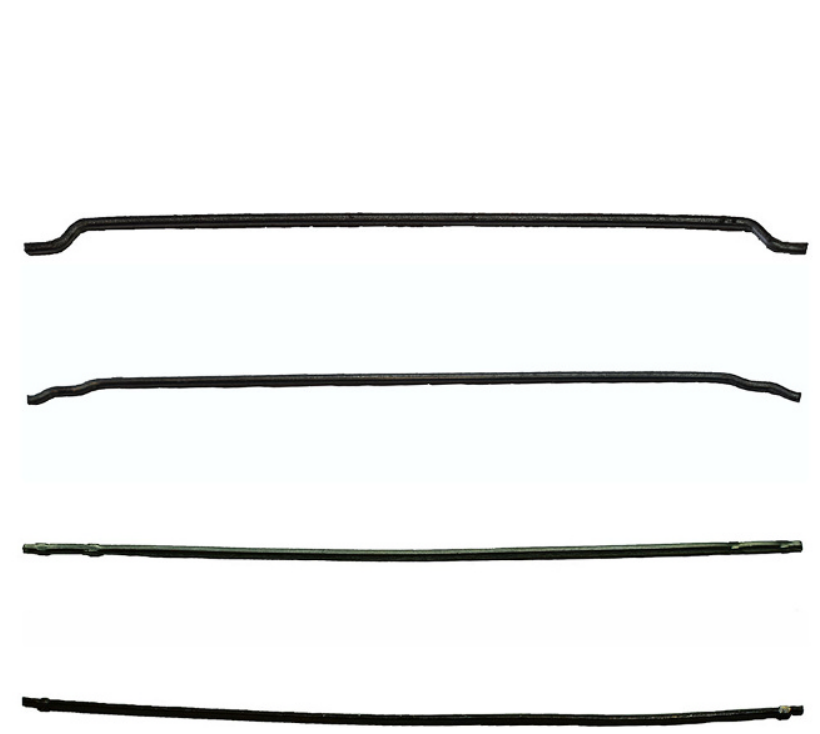

Abb. 4: Von oben:3D-Stahlfaser, 4D-Stahlfaser, Stahlfaser mitzwei Endknoten, Stahlfaser mit einem Endknoten

für die Bemessung durch Multiplikation mit einer Konstanten von 0,37 auf die direkte Zugfestigkeit $f_{\text {fku }}$ umgerechnet und für den Designwert $f_{f d u}$ durch den Sicherheitsfaktor für Beton laut EC 2 [5] von 1,5 dividiert. Als Modell für die Bemessung dient ein linear-elastischer Ansatz auf Druck und ein perfekt-plastisches Materialverhalten auf Zug. Die Zugzonenhöhe beträgt $90 \%$ der Bauteilhöhe $\mathrm{h}$ und der innere Hebelarm wird mit der Hälfte der Bauteilhöhe $h$ angenommen.

Der in der ÖVBB-RL „Tübbingsysteme aus Beton“ [3] geforderte Nachweis der Rissweite für den Grenzzustand der Gebrauchstauglichkeit ist in der Richtlinie „Faserbeton“ [4] nicht enthalten. Das Fehlen dieses Nachweises ergibt zwischen den beiden Richtlinien einen Widerspruch und somit ist eine Anwendung in Österreich zurzeit nicht möglich.

Zusätzlich ergibt die beschriebene Vorgehensweise für die Auswertung der Biegezugversuche und die Bemessung des Bauteils im Maßstab 1:1 Divergenzen, da für ein und denselben Werkstoff bei gleichartiger Belastung (Biegung) zwei unterschiedliche Materialverhalten zugrunde gelegt werden. Bei der Auswertung der Biegebalkenversuche wird auch für den gerissenen Bereich ein linear-elastisches Ma- 


\begin{tabular}{|lllll}
\hline $\begin{array}{l}\text { TABELLE } \\
\text { Gegenüberstellung Fasern }\end{array}$ & & \\
\hline Serie & Fasertyp/Endverankerung & Fasergehalt, $\mathrm{kg} / \mathrm{m}^{3}$ & Schlankheit & Zugfestigkeit, MPa \\
\hline 1 & 3D & 40 & 60 & 2400 \\
\hline 2 & 4D & 50 & 80 & 1500 \\
\hline 3 & Doppelter Endknoten & 50 & 80 & 2050 \\
\hline 4 & Einfacher Endknoten & 60 & 80 & 2000 \\
\hline
\end{tabular}

terialverhalten über die ganze Balkenhöhe verwendet, wohingegen bei der Bemessung des Bauteils in diesem Zustand ein perfekt-plastisches Materialverhalten unter Zugbelastung vorausgesetzt wird.

Die hierbei angesetzte direkte Zugfestigkeit für die Bemessung im Grenzzustand der Tragfähigkeit wird, wie beschrieben, über konstante Faktoren aus den Biegebalken ermittelt. Aufgrund der großen Streuungen der Versuchsergebnisse werden diese Werte stark abgemindert und nur ca. 1/8 der mittleren Biegezugfestigkeit wird für die Bemessung als Designwert der direkten Zugfestigkeit im Grenzzustand der Tragfähigkeit angesetzt. Dies führt folglich dazu, dass der Nachweis der Rissbreitenbeschränkung im Grenzzustand der Gebrauchstauglichkeit gar nicht erbracht werden kann, da die Werte noch stärker abzumindern sind.

\section{Versuche}

Aufgrund der beschriebenen Diskrepanzen in der Bemessung, aber einer Forderung des Einsatzes von Faserbeton durch die Industrie wurden erste Versuche zur Ermittlung des Trag- und Verformungsverhaltens von Stahlfasertübbingen durchgeführt.

Mithilfe des in Kooperation zwischen ÖBB-Infrastruktur AG und Montanuniversität Leoben - Lehrstuhl für Subsurface Engineering entstandenen, servo-hydraulischem Tübbingprüfstands (Abb. 3) können biaxiale Versuche an Tübbingen mit realen Geometrien durchgeführt werden [1]. An der Horizontalachse können Kräfte von insgesamt $4 \mathrm{MN}$ und an der Vertikalachse von $2 \mathrm{MN}$ aufgebracht werden. Je nach Anforderungen an den zu untersuchenden Tübbing wird das Segment mit unterschiedlichen Belastungskollektiven mithilfe der integrierten SPS belastet. Als Standardmesssystem haben sich, wie schon bei den konventionell bewehrten Segmenten, Kraftmessdosen an jedem Hydraulikzylinder, Seilzugsensoren zur Verschiebungsmessung und Dehnungsmessstreifen zur Risserkennung und -detektion bei den durchgeführten Versuchen bewährt.

Wie schon bei den Versuchen mit stabbewehrten Tübbingen wurden die Faserbetontübbinge am Koralmtunnel Baulos KAT 2 hergestellt, kommen aber aufgrund des aktuellen Stands der Regelwerke und Richtlinien dort nicht zum regulären Einsatz und wurden nur für Forschungszwecke produziert. Hierbei wurden vier Serien mit jeweils zwei bis drei Segmenten mit unterschiedlichen Fasern von verschiedenen Herstellern eingesetzt. Für die ersten drei Versuchsserien wurde eine gleichbleibende Betonrezeptur der Betongüte C35/45 verwendet. Zur Optimierung des Erstrissverhaltens wurde aufgrund der Vorkenntnisse aus den stabbewehrten Segmenten für die vierte Serie ein Beton der Güte C50/60 herangezogen. Als Anforderung an die Faserbetontübbinge wurde ein Trag- und Verformungsverhalten zumindest des Normaltübbings sowie ein duktiles Verhalten im Nachrissbereich gestellt.

\section{Stahlfasertypen}

Bei den eingesetzten Stahlfasern handelte es sich ausschließlich um statisch wirksame Makrofasern mit einer Länge von $60 \mathrm{~mm}$, welche im Mischvorgang dem Beton zugegeben wurden. Diese Fasern unterscheiden sich hinsichtlich ihrer Endverankerung (Abb. 4), Zugfestigkeit und Schlankheit, dem Verhältnis von Faserlänge zu Nenndurchmesser (Tab. 1).

\section{Ergebnisse}

Im Folgenden wird auf Ergebnisse von Tübbingversuchen mit Belastungskollektiven ähnlich Dreipunktbiegeversuchen mit horizontaler Stützung eingegangen. Hierbei erfolgte die Lastaufbringung nach einer Grundstufe, bei der die Horizontal- und Vertikalkraft gleichmäßig bis ca. $80 \mathrm{kN}$ aufgebracht wurden, nur noch durch Erhöhung der Vertikalkraft in Laststufen von 10 bis $25 \mathrm{kN}$. Alle Versuche mit Faserbeton hatten gemeinsam, dass das Versagen des Bauteils durch einen maßgebenden Hauptriss in der Tübbingmitte stattfand. Die visuelle Beurteilung der Bruchflächen nach der Versuchsdurchführung hat ergeben, dass die Stahlfasern bei allen durchgeführten Versuchen vorwiegend aus der Betonmatrix ausgezogen wurden.

Der idente Fasergehalt sowie die gleiche Betongüte und das Ergebnis der Vorversuche anhand von Dreipunktbiegeversuchen nach EN 14651 ([5]; Abb. 5) lässt vermuten, dass die Großversuche an Tübbingen gleichartige Ergebnisse lieferten. In Abb. 6 ist die Zugspannung $\sigma$ an der Hohlraumseite (Intrados), welche mittels der aufgebrachten Kräfte, der Geometrie des Segments und einem linearelastischen Ansatz ermittelt wurde, über die relative Vertikalverschiebung in der Tübbingmitte (1.3 und 2.3) für die Versuche der zweiten (V17-V19) und dritten Serie (V20, V21) gegenübergestellt. Anzumerken ist, dass die berechnete Zugspannung $\sigma$ am Intrados nach dem Auftreten des Erstrisses keine physikalisch-mechanische Größe darstellt und nur für den Vergleichszweck der biaxialen Versuche herangezogen wird. Somit werden nach diesem die Ergebniskurven strichliert dargestellt. Im Diagramm ist ersichtlich, dass der Erstriss unabhängig vom Fasertyp im gleichen Werte- 
Abb. 5: Biegezugfestigkeiten und residuelle Biegezugfestigkeiten ermittelt mittels Dreipunktbiegeversuchen nach EN 14651 [5] für die Serien 2 und 3 mit Standardabweichung [2]

Abb. 6: Vergleich der Versuche der zweiten (V17-V19) und dritten Serie (V20, V21) [2]
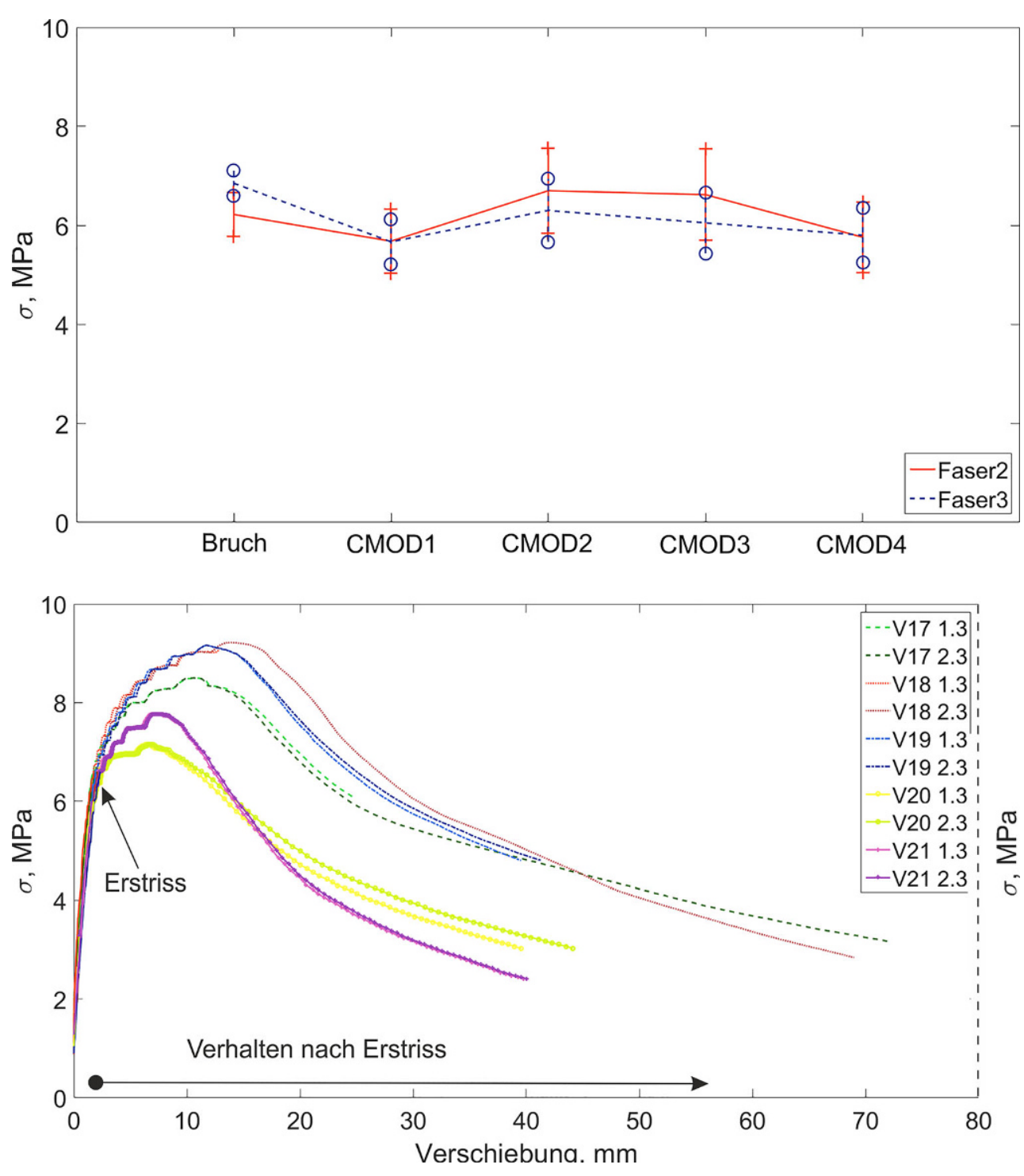

bereich auftrat, das Tragverhalten nach dem Erstriss sich aber deutlich unterschied. Dieses Ergebnis ist insofern erstaunlich, da wie bereits beschrieben, die durchgeführten Dreipunktbiegeversuche nach EN 14651 [5] mit $50 \mathrm{~kg} / \mathrm{m}^{3}$ der beiden Fasertypen sowie einer Betongüte C35/45 quasi idente Ergebnisse lieferten. Aufgrund dieser Erkenntnisse ist die Anwendbarkeit von Drei- bzw. Vierpunktbiegeversuchen zur Parametergewinnung und Betoncharakterisierung für statisch beanspruchte Großbauteile wie Tübbinge zu hinterfragen.

Im Diagramm der Abb. 7 sind die Versuche der zweiten (V17-V19) dem Versuch 23 der vierten Serie gegenübergestellt. Das zweite Segment der vierten Serie (V22) wurde aufgrund eines stark außermittigen Risses nicht für den Vergleich herangezogen. Wie bereits erwähnt, wurden für die dritte Serie $50 \mathrm{~kg} / \mathrm{m}^{3}$ Stahlfasern und eine Betongüte C35/45 und für die vierte Serie $60 \mathrm{~kg} / \mathrm{m}^{3}$ Stahlfasern und eine Betongüte $\mathrm{C} 50 / 60$ eingesetzt. Hierbei ist ersichtlich, dass trotz höherem Fasergehalt und höherer nomineller Betongüte der Versuch 23 eine geringere Resttragfähigkeit aufwies.
Aus diesem Vergleich wird deutlich, dass für die Spezifikation von Faserbeton der Fasergehalt und die Betongüte alleine keine Aussage über das Tragverhalten zulassen.

Der Vergleich aller durchgeführten Versuche mit Stahlfaserbeton (SF) mit einem Normaltübbing (V16) mit reiner Lastfallbewehrung (Abb. 8) zeigt, dass Stahlfaserbeton eine Alternative zu konventioneller Stabbewehrung vor allem in Gebirgsbereichen mit geringer Momentenbelastung des Tübbingausbaus darstellt. Schon $40 \mathrm{~kg} / \mathrm{m}^{3}$ Stahlfasern führten zu dem gefordertem duktilen Verhalten nach dem Auftreten des Erstrisses und erfüllten somit die gesetzten Anforderungen.

Zusätzlich zu diesen Erkenntnissen wurde bei der Nachrechnung der Versuche mittels der Parameter aus den begleitenden Laborversuchen und dem in der ÖVBB-RL „Faserbeton" [4] gegebenen Formelwerk nicht akzeptable Diskrepanzen festgestellt. Mit diesem Bemessungsmodell ist die Bestimmung des Trag- und Rissverhaltens der untersuchten Tübbinge nicht möglich. Darüber hinaus ist der rechnerische Nachweis der Spaltzugtragfähigkeit in den 
Abb. 7: Vergleich der Versuche der zweiten (V17-V19) und vierten Serie (V23) [2]

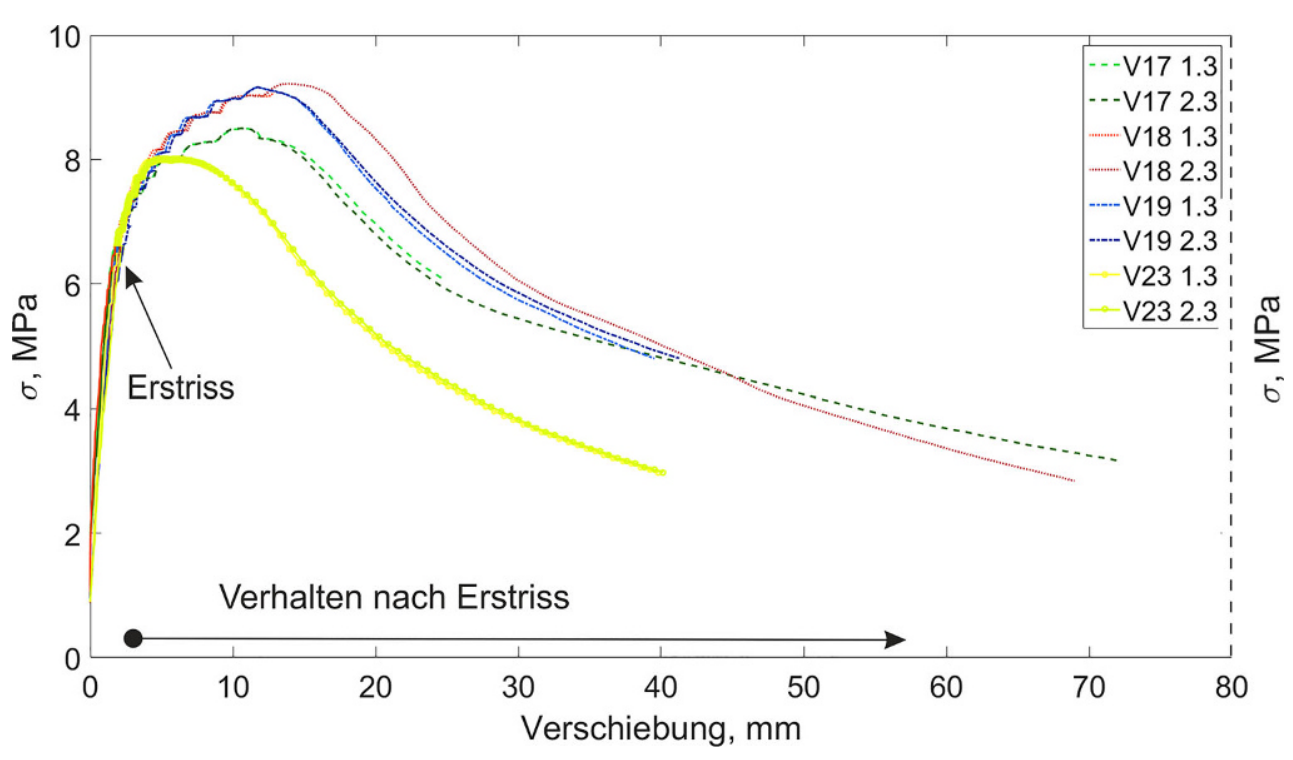

Abb. 8: Vergleich aller Stahlfaserbetonversuche mit einem Normaltübbing (V16) [2]

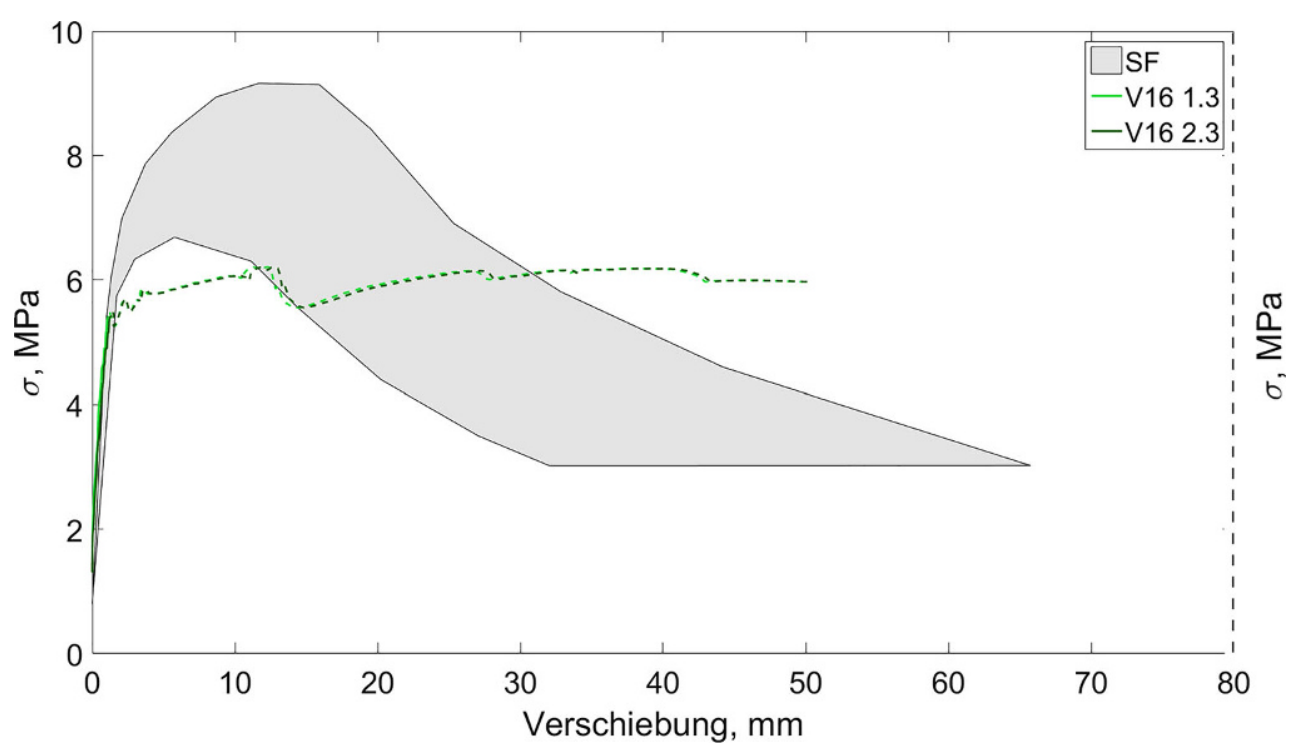

Längs- und Ringfugen, basierend auf gegebenen Belastungen aus der Tübbingstatik [6], mit reinem Faserbeton nicht zu erbringen. Dies liegt einerseits an den sehr konservativen Annahmen der Nachbruchzugfestigkeit des Faserbetons sowie auch an der Parametergewinnung durch die stark vereinfachte Auswertung der durchgeführten Biegeund indirekten Zugversuche.

\section{Schlussfolgerung und Ausblick}

Die durchgeführten Versuche mit Stahlfasersegmenten haben gezeigt, dass dieser Werkstoff in Zukunft durchaus eine Option zum Ersatz des Bewehrungskorbs bei der Produktion von Normaltübbingen darstellt. Durch das Fehlen von geeigneten Versuchen zur Parameterermittlung sowie die aufgezeigten Diskrepanzen in den Regelwerken zur Dimensionierung dieser Bauteile ist die Verwendung in der Praxis in Österreich aktuell de facto nicht möglich. Daher arbeitet der Lehrstuhl für Subsurface Engineering aktuell in Kooperation mit Forschungs- und Industriepartnern an einem Projekt zur Überarbeitung bzw. Ergänzung der vorhandenen Regelwerke. Hierbei soll besonders auf neue Methoden zur Materialcharakterisierung, die Optimierung des Mischvorgangs und die Erstellung neuer Rechenmodelle zur Dimensionierung eingegangen werden.

Funding. Open access funding provided by Montanuniversität Leoben.

Open Access Dieser Artikel wird unter der Creative Commons Namensnennung 4.0 International Lizenz (http://creativecommons.org/licenses/ by/4.0/deed.de) veröffentlicht, welche die Nutzung, Vervielfältigung, Bearbeitung, Verbreitung und Wiedergabe in jeglichem Medium und Format erlaubt, sofern Sie den/die ursprünglichen Autor(en) und die Quelle ordnungsgemäß nennen, einen Linkzur Creative Commons Lizenz beifügen und angeben, ob Änderungen vorgenommen wurden. 


\section{Literatur}

1. Gehwolf, P.; Wenighofer, R.; Barwart, S.; Galler, R.; Haberler-Weber, M.; Moritz, B.; Barwart, C.; Lange, A.: Konzeptionierung, Realisierung und erste Ergebnisse eines neu entwickelten Tübbingprüf stands, BHM Berg- und Hüttenmännische Monatshefte 160 (2015), H. 12 , S. 530-534

2. Gehwolf, P.: Tragverhalten von Tübbingen im maschinellen Tunnelbau - Experimentelle und numerische Analyse, Diss., Leoben, Montanuniversität, Lehrst. für Subsurface Engineering, 2018
3. Österreichische Vereinigung für Beton- und Bautechnik: Richtlinie Tübbingsysteme aus Beton, 2009

4. Österreichische Vereinigung für Beton- und Bautechnik: Richtlinie Faserbeton, 2008

5. ÖNORM EN 14651: Prüfverfahren für Beton mit metallischen Fasern - Bestimmung der Biegezugfestigkeit, 2007

6. ARGE Koralmtunnel - KAT2: Tübbingdesign - Statik, Teil 1 - Grundlagen, Allgemeine Beschreibung und Nachweise, unveröffentlicht, 2011 